\title{
Observational challenges for the standard FLRW model
}

\author{
Thomas Buchert ${ }^{1}$, Alan A. Coley ${ }^{2}$, Hagen Kleinert ${ }^{3}$, Boudewijn F. Roukema ${ }^{4,1}$ \\ and David L. Wiltshire ${ }^{5}$ \\ ${ }^{1}$ Université de Lyon, Observatoire de Lyon, Centre de Recherche Astrophysique de Lyon, \\ CNRS UMR 5574: Université Lyon 1 and École Normale Supérieure de Lyon, \\ 9 avenue Charles André, F-69230 Saint-Genis-Laval, France* \\ ${ }^{2}$ Department of Mathematics and Statistics, Dalhousie University, \\ Halifax, NS B3H 3J5, Canada \\ ${ }^{3}$ Institute for Theoretical Physics, Freie Universität Berlin, \\ Arnimallee 14, 14195 Berlin, Germany \\ ${ }^{4}$ Toruń Centre for Astronomy, Faculty of Physics, Astronomy and Informatics, Grudziadzka 5, \\ Nicolaus Copernicus University, ul. Gagarina 11, 87-100 Toruń, Poland \\ ${ }^{5}$ Department of Physics and Astronomy, University of Canterbury, \\ Private Bag 4800, Christchurch 8140, New Zealand
}

\begin{abstract}
We summarise some of the main observational challenges for the standard FriedmannLemaitre-Robertson-Walker cosmological model and describe how results recently presented in the parallel session "Large-scale Structure and Statistics" (DE3) at the "Fourteenth Marcel Grossman Meeting on General Relativity" are related to these challenges.

Keywords: large-scale structure; cosmic microwave background; statistics; generalrelativistic effects

PACS numbers: 98.80.-k, 98.80.Es, 98.80.Jk, 95.36.+x, 04.20.-q, 04.40.-b
\end{abstract}

\section{Observational Challenges for the $\Lambda \mathrm{CDM}$ Model}

Despite the many well-known successes of the FLRW model with its standard parameter values, henceforth denoted the $\Lambda \mathrm{CDM}$ model, there is a wide range of observations with which it significantly disagrees. The statistical significance of these disagreements is very often debated from a Bayesian perspective. If the $\Lambda$ CDM is accepted as being consistent with general relativity, then one must contend with $a$ posteriori statistics, also called the look elsewhere effect, which globally requires a Šidàk-Bonferonni correction [1] for assessing overall statistical significance. On the other hand, interpretation of structure formation within the $\Lambda \mathrm{CDM}$ model is to a large degree based on Newtonian physics - $N$-body simulations are widely seen as providing state-of-the-art ways of comparing the FLRW model to observational catalogues - but in comparison to general relativity, the former should be assigned an extremely weak prior. ${ }^{a}$ Although precise tests of general relativity have led to

*BFR: During invited lectureship.

${ }^{a}$ For example, Keplerian orbits are disfavoured in relation to general-relativistic orbits at a significance level of more than $100 \sigma$ [2] based on the periastron decay of the Hulse-Taylor pulsar B1913+16 [3] (See Fig. 2 of Ref. 2.) 
overwhelming Bayesian evidence vis-à-vis Newtonian gravity for binary systems on small scales, it could be argued that one cannot extrapolate any Bayesian comparison of the two theories to cosmological scales. However, there are no fundamental reasons to prefer Newtonian gravity over full general relativity on such scales, given its intrinsic theoretical shortcomings of absolute space and time and action-at-adistance, redundant concepts which are more problematic as the scales grow larger. We will not attempt a full Bayesian analysis, which would require us to assign relative merits to full general relativity versus the FLRW model plus inhomogeneities obeying Newtonian gravity. Rather we will focus on individual observational constraints.

One observational contradiction with $\Lambda \mathrm{CDM}$ that is widely accepted in the community is the primordial lithium abundance anomaly: the lack of ${ }^{7} \mathrm{Li} / \mathrm{H}$ in metal-poor halo field stars in our Galaxy is inconsistent with the $\Lambda$ CDM cosmic

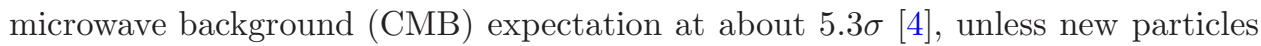
such as decaying gravitinos are assumed [5].

While other difficulties for the $\Lambda \mathrm{CDM}$ model from the CMB - the large-angle anomalies - are more often described as "tensions", these disagreements with the model are numerous and their statistical significance has tended to increase or remain stable as the accuracy and precision of the data have increased $[6,7]$. At the largest angular scales there is a lack of power, which translates to a lack of observed structure in the CMB with respect to $\Lambda \mathrm{CDM}$ on the largest scales. This was suspected in the COsmic microwave Background Explorer (COBE) maps, detected by the Wilkinson Microwave Anisotropy Probe (WMAP), and confirmed by the Planck Surveyor first data [8-11]. This observation means that the observed Universe is too (spatially) homogeneous on the largest $\left(>10 h^{-1} \mathrm{Gpc}\right)$ scales, as measured by $S_{1 / 2}$, the integral of the squared auto-correlation of cosmological CMB temperaturetemperature fluctuations on angular scales greater than $60^{\circ}$ or spatial scales greater than the radius to the surface of last scattering. [11] One of the proposed explanations is that a finite universe model (without a spatial boundary) naturally explains this lack of power $[8,12-20]$. In terms of spherical harmonic decomposition of the sky maps, this is not restricted to only the quadrupole signal [9-11]. Figure 3 and Table 2 in Ref. 11 show why the Planck data lead to rejection of the $\Lambda$ CDM model with probability $p<0.0024$ for conservative versions of the data, or $p<0.0003$ for the best quality data. Hemispherical asymmetry rejecting the $\Lambda \mathrm{CDM}$ model at about the $3 \sigma$ level has remained a stable problem since it was first detected in WMAP [21-24]. Low spherical harmonic mode number $l$ alignments in the CMB also remain inconsistent with $\Lambda \mathrm{CDM}$ at about the $3 \sigma$ level in the Planck data $[25,26]$.

Comparison of the integrated Sachs-Wolfe (ISW) effect in the CMB (WMAP5) to stacked foreground voids, using luminous red galaxies (LRGs), from the Sloan Digital Sky Survey Data Release 6 (SDSS DR6), gives a temperature decrement $\Delta T_{\mathrm{ISW}}=9.6 \pm 2.2 \mu \mathrm{K}$, which is significantly (at least $3 \sigma$ ) greater than the $\Lambda \mathrm{CDM}$ expected value of $2.27 \pm 0.14 \mu \mathrm{K}[27,28]$. Independently of the CMB, Minkowski functionals of LRG-traced structure at scales of tens of megaparsecs in a volume 
of diameter $500 h^{-1} \mathrm{Mpc}$ in SDSS DR7 are also inconsistent with $\Lambda \mathrm{CDM}$ at about $3 \sigma[29]$.

Combining many measurements at different scales, a $5 \sigma$ contradiction is found between the standard $\Lambda \mathrm{CDM}$ model and observations, mainly due to the differing power in the small-scale and large-scale parts of the flat-space power spectrum $P(k)$ [30]. Comparison of low-redshift weak gravitational lensing of galaxies in the Canada France Hawaii (Telescope) Lensing Survey (CFHTLenS) to CMB constraints, interpreted according to $\Lambda \mathrm{CDM}$, leads to a present-day matter density parameter $\Omega_{\mathrm{m} 0}$ which is too low in the $\sigma_{8}-\Omega_{\mathrm{m} 0}$ plane (where $\sigma_{8}$ is the root-meansquare density fluctuation in a sphere of radius $8 h^{-1} \mathrm{Mpc}$ and the Hubble constant is $H_{0}=100 \mathrm{hm} \mathrm{s}^{-1} \mathrm{Mpc}^{-1}$ ), at a rejection level of $p \leq 0.1$, unless sterile neutrinos are added to the model [31].

The Baryon Acoustic Oscillation (BAO) signal of Lyman $\alpha$ forest absorbers in front of quasars measured in the SDSS III/Baryon Oscillation Spectroscopic Survey (BOSS)/Data Release 11 contradicts the $\Lambda$ CDM expectation at about 2.5 $\sigma$ [32]. The normalised growth rate dependence on redshift $f \sigma_{8}(z)$, as measured in BOSS and the WiggleZ Dark Energy Survey (WiggleZ) [33] is about $2 \sigma$ too low in comparison to the Planck $\Lambda$ CDM expectation. (See Fig. 6 in Ref. 34.) A Bayesian multi-survey analysis comparing $\mathrm{CMB}$, supernovae type Ia data and galaxy surveys finds that the probability of the $\Lambda \mathrm{CDM}$ model being correct is $p \leq 0.01$ [35].

There may be a $\Lambda \mathrm{CDM}$ inconsistency with an overabundance of luminous starforming galaxies of $10^{8} M_{\odot}$ forming too early, at $z \approx 10$, as indicated by the number of Lyman break galaxies (LBGs) detected so far using the Hubble and Spitzer Space Telescopes [36]. Furthermore, the dark matter side of $N$-body simulations in the $\Lambda \mathrm{CDM}$ cosmology is not able to readily account for a number of the detailed features observed in the Local Group of galaxies [37], including the numbers, densities and spatial distribution of dwarf satellite galaxies, in addition to a general overabundance of halos in simulations as compared with what is observed.

\section{Observational tests of the FLRW geometry}

Many results of the standard cosmology are based on very large $N$-body numerical simulations using Newtonian gravity on a FLRW background. While such simulations yield values of the Newtonian potential in the present Universe no larger than $10^{-4}$, they presuppose the validity of the FLRW geometry. Model-independent observational tests of the validity of the FLRW geometry are therefore particularly important.

Such tests can be split into two classes: (i) tests of the validity of the Friedmann equations over long epochs of cosmic history in the regime where average Hubble expansion variation is nonlinear; (ii) tests of the validity of the spatial FLRW geometry below the scale of statistical homogeneity. (Hereafter, as in common usage, when stating "homogeneity", we refer to spatial homogeneity, unless otherwise stated.) 


\subsection{Tests of the Friedmann equation}

The violation of the FLRW relationship between the average expansion rate and the luminosity distance can be used as a test of the importance of inhomogeneities [38-47]. This is exemplified by the Clarkson-Bassett-Lu (CBL) test [38]: the spatial curvature parameter of the FLRW model, a constant, may be written as

$$
\widehat{\Omega}_{k}=\frac{\left[H(z) D^{\prime}(z)\right]^{2}-1}{\left[H_{0} D(z)\right]^{2}}
$$

for all redshifts, where $H(z)$ is the Hubble parameter, $H_{0}=H(0)$ the Hubble constant, and $D(z)=d_{\mathrm{L}} /(1+z)$ where $d_{\mathrm{L}}$ is the luminosity distance to a redshift $z$. This test assumes that $H$ and $D$ depend on $z$ alone. A further derivative of (1) with respect to $z$ then gives a statistic which must be identically zero for all redshifts if the Friedmann equations apply, irrespective of any dark energy model or alternative model parameters. Model-independent observations of $H(z), D(z)$ and $D^{\prime}(z)$ for sufficiently large data sets can therefore be a powerful discriminator of the standard cosmology from inhomogeneous cosmological models, such as those with backreaction from inhomogeneities or exact inhomogeneous solutions. Inhomogeneous cosmological models do not, in general, obey the Friedmann equation at late epochs and do not have a uniform spatial curvature, so that (1) is not constant with redshift. Predictions have been made for specific models which incorporate backreaction $[39,40,45,46]$.

\subsection{The scale of statistical homogeneity}

Many debates about the role of inhomogeneities in observational challenges to the standard model involve different approaches to the definition of a scale of statistical homogeneity. Generally one must deal with spatial averages of the density field, defined on a compact domain of a spatial hypersurface, $\Sigma_{t}$, according to

$$
\langle\rho(t)\rangle_{\mathcal{D}_{R}}=\frac{1}{\mathcal{V}(t)}\left(\int_{\mathcal{D}_{R}} \mathrm{~d}^{3} x \sqrt{\operatorname{det}^{3} g} \rho(t, \mathbf{x})\right),
$$

where $\mathcal{V}(t) \equiv \alpha R^{3}(t)=\int_{\mathcal{D}_{R}} \mathrm{~d}^{3} x \sqrt{\operatorname{det}^{3} g}$ is the volume of the domain $\mathcal{D}_{R} \subset \Sigma_{t}$, $g_{i j},(1 \leq i, j \leq 3)$ is the intrinsic metric on $\Sigma_{t}$ and $\alpha$ is a dimensionless constant determined by a choice of geometry; e.g., $\alpha=4 \pi / 3$ for Euclidean spheres. In approaches in which ergodicity is assumed to apply, a definition of homogeneity often presupposes the existence of an average positive density, $\rho_{0}(t)$, defined by the limit

$$
\lim _{R(t) \rightarrow \infty}\langle\rho(t)\rangle_{\mathcal{D}_{R}}=\rho_{0}(t)>0 .
$$

A homogeneity scale, $\lambda_{0}(t)$, is then defined [48] by the requirement that every point in $\Sigma_{t}$ be contained in a domain $\mathcal{D}_{\lambda_{0}} \subset \mathcal{D}_{R}$ such that

$$
\left|\langle\rho(t)\rangle_{\mathcal{D}_{R}}-\rho_{0}(t)\right|<\rho_{0}(t) \quad \forall R>\lambda_{0} .
$$

In practice, the density field can only be inferred indirectly from the statistical properties of the distribution of galaxies, with all of the systematic issues related 
to finite sample volumes and observational biases. Thus, any practical measure of statistical homogeneity is not directly based on a relation such as (4), but rather on the scale dependence of galaxy-galaxy correlation functions.

Observationally, the range $70-120 h^{-1} \mathrm{Mpc}$ comprises the smallest scales at which any notion of statistical homogeneity can be argued to emerge $[49,50]$ at the present epoch, based on the 2-point correlation function. However, if all $N$-point correlations of the galaxy distribution are taken into account, then the homogeneity scale is expected to be reached, if at all, only on scales beyond $700 h^{-1} \mathrm{Mpc}$ [29]. Improved survey quality (distance, volume and methods) tends to have increased estimates of the homogeneity scale over the last few decades, e.g., see Ref. 51. Another example is reported in Refs. 52,53 : the $\approx 105 h^{-1} \mathrm{Mpc}$ BAO scale, normally thought of as a comoving standard ruler, is shrunk from about $6 \%$ to $10 \%$ across SDSS DR7 superclusters, as a function of increasing overlap between luminous red galaxy (LRG) pair paths and superclusters (assuming a $\Lambda$ CDM model when interpreting the observations). Writing the metric near a supercluster using one of the standard FLRW expressions, but replacing homogeneous parameters by effective parameters, this represents a deviation in the spatial metric coefficients, $g_{i j}$, of $6-10 \%$.

Estimates of the present epoch variation of the density on the largest possible scales in SDSS DR7, limited only by survey volumes ${ }^{\mathrm{b}}$, have given a standard deviation of order [54] $8 \%$, consistent with an earlier measurement of $7 \%$ in a smaller sample [49]. In the $\Lambda \mathrm{CDM}$ cosmology the understanding of this standard deviation is subject to the observational interpretation of cosmic variance arising from the evolution of initial density perturbations, given that the assumption of ergodicity will not hold on every assumed large scale. Hence, the amplitude of (4) on large scales does not itself provide a model-independent test of the FLRW geometry.

\subsection{Tests of spatial geometry on "small" scales}

Even if one accepts the most conservative estimate of $70-120 h^{-1} \mathrm{Mpc}$ as the scale at which some notion of statistical homogeneity emerges, with an effective FLRW geometry at larger scales, then the assumption that such a geometry applies at smaller scales is no more than a working hypothesis ${ }^{\mathrm{c}}$ which must be seriously questioned given that the largest typical inhomogeneities are voids of diameter $\sim 30 h^{-1} \mathrm{Mpc}$

\footnotetext{
${ }^{\mathrm{b}}$ Specifically, Sylos Labini et al [54] divided the full sample of 53,066 luminous red galaxies in the redshift range $10^{-4}<z<0.3$ into $N$ equal nonoverlapping volumes. The standard deviation of order $8 \%$ is found for the range $4 \leq N \leq 15$.

${ }^{c}$ It is sometimes claimed that the FLRW metric is applicable on all scales, "except in the immediate vicinity of black holes and neutron stars" [55]. However, such statements merely reflect the assumptions of the standard cosmological model, rather than direct observational facts or general mathematical theorems. In view of the observational results reviewed here, we know that the assumptions made in Refs. 55, 56 are not applicable to the real physical Universe. A critique of the mathematical results of Refs. 55, 56 is given in Ref. 57, and is discussed further in Ref. 58.
} 
and density contrast $\delta \rho / \rho \sim-0.95$, which form some $40 \%$ of the volume ${ }^{\mathrm{d}}$ of the Universe at low redshifts $[63,64]$. Density variations of order $100 \%$ are thus guaranteed when smoothing on scales of tens of megaparsecs, and constitute a regime which is accepted as nonlinear in the standard cosmological model. This regime is commonly treated by Newtonian $N$-body simulations, leading to many phenomenologically realistic results. However, given that the regime is nonlinear then the first principles of general relativity, which demand a coupling of matter and geometry, have no $a$ priori preference for the FLRW geometry modified by Newtonian gravity on these scales. This is why there have been several recent attempts to construct relativistic simulations [65-68], some of which are summarised in Ref. 69.

Furthermore, the hypothesis of applicability of the FLRW spatial geometry on scales $\lesssim 100 h^{-1} \mathrm{Mpc}$ is open to direct observational test. If the hypothesis were true, then all motions of galaxy clusters - the largest bound structures - should reduce to a uniform FLRW expansion plus local Lorentz boosts, in contrast to general inhomogeneous solutions of Einstein's equations which exhibit a differential expansion of space. If all departures from homogeneity are described by local boosts on an FLRW geometry, then the dipole anisotropy in the CMB is purely kinematic, as is conventionally assumed.

In addition to the known motions of the Sun in our galaxy, and our galaxy's motion within the Local Group of galaxies, the kinematic interpretation of the CMB dipole requires that the local group is boosted at $635 \pm 38 \mathrm{~km} \mathrm{~s}^{-1}$ in a direction $(\ell, b)=\left(276.4^{\circ}, 29.3^{\circ}\right) \pm 3.2^{\circ}[70]$. However, decades of work by astronomers to explain the amplitude of this "clustering dipole" has led to an ongoing debate about the convergence of bulk flows on scales $\lesssim 120 h^{-1} \mathrm{Mpc}[71,72]$. Very recently, a resolution of this debate has been claimed in Ref. 73 on the basis of constrained $N$-body simulations, which incorporate various technical improvements such as estimates for uncertainties associated with missing attractors. However, given the complexities of the statistical interpretation of numerical simulations - e.g., the definition of what the peculiar velocity is on a sample that is considerably smaller than the homogeneity scale - and the $20 \%$ uncertainty in the magnitude of the final result in Ref. 73 , it is likely that the debate will continue.

In contrast, a recent purely observational model-independent analysis [74] of the COMPOSITE sample of 4,534 group and cluster distances found that the spherically averaged Hubble expansion is very significantly more uniform in the rest frame of the Local Group (LG) as compared to the standard rest frame of the $\mathrm{CMB}^{\mathrm{e}}$ (The

\footnotetext{
${ }^{\mathrm{d}}$ The overall statistics [59] include a small fraction of larger voids and a large population of smaller minivoids [60], making the Universe void dominated at the present epoch with typical theoretical estimates of the void filling-fraction of up to $80 \%$ [61,62]. By volume the Universe is mostly empty, with a very spiky density distribution in the remaining tiny volumes.

${ }^{\mathrm{e}} \mathrm{A}$ further search for a rest frame of minimum nonlinear Hubble expansion variation [75] finds that the LG frame is consistent with such a frame but the statistical likelihood is not changed significantly under local boosts within the plane of our galaxy on account of a lack of constraining data in the region obscured by the Milky Way. Such degenerate boost directions do not include
} 
Bayesian evidence is very strong, with $\ln B>5$.) While this result is completely unexpected if the clustering dipole is purely kinematic, the residual monopole and dipole variation of the Hubble expansion in the Local Group frame was found to be consistent with a $0.5 \%$ anisotropy in the distance-redshift relation on $\lesssim 65 h^{-1} \mathrm{Mpc}$ scales [74], an effect which can be reproduced using nonlinear exact solutions of Einstein's equations with inhomogeneities no larger than the scales in question [76].

Although the Planck Surveyor team claims to have measured Lorentz boosting through the effects of frequency modulation and aberration on the angular power spectrum, the boost direction is consistent with that of the CMB dipole only when small angular scales are considered [77]. When large angle multipoles are included the dipole moves across the sky to point in the modulation dipole anomaly direction [22]. The scale-dependence of this result in Ref. 77 therefore suggests an intriguing possibility that certain large-angle anomalies in the CMB power spectrum may arise from treating a partly nonkinematic dipole purely kinematically; a direct result of the relevant geometry being non-FLRW. In an independent study, the hypothesis of a purely kinematic origin for the dipole in the cosmic distribution of radio galaxies has been rejected at the $99.5 \%$ confidence level [78], using the NRAO VLA Sky Survey (NVSS).

\section{Recent developments presented at the MG14 meeting}

Recent work in statistical assessment of large-scale structure was presented at the "Fourteenth Marcel Grossman Meeting on General Relativity" (MG14) parallel session DE3, "Large-scale Structure and Statistics". This included higher-order statistics, morphological properties, the reality and significance of large structures in the Universe, standard rulers like the baryon acoustic oscillation (BAO) peak location, comparisons with mock catalogues, path finders for next-generation galaxy catalogues, non-Gaussian statistics, mass functions and abundance statistics of collapsed objects, among other topics. Contributions also included general-relativistic aspects of large-scale structure formation in these contexts, such as the measurement of backreaction in large-scale structure data, indirect measures of metrical properties and improved redshift-distance measures in inhomogeneous cosmologies. The session was divided into observational issues and modelling aspects. In Sect. 4 we mainly concentrate on the former, by looking at various observational cornerstones and highlighting current challenges to the standard Friedmann-LemaitreRobertson-Walker (FLRW) model and those likely to be faced in the near future. Marco Bruni's report briefly summarises modelling work that was contributed to the parallel session [69].

Theoretical issues, including backreaction and inhomogeneous cosmology, were discussed in another MG14 parallel session, DE2. Possible observational challenges

that from the LG to CMB rest frame, however. 
to the standard FLRW model ${ }^{\mathrm{f}}$ and theoretical discussion of the effects of inhomogeneities were discussed in the individual talks of both the DE2 and DE3 sessions and during a special discussion session. (See, in particular, the summary of Ostrowski's presentation [58].) A statistical question that does not yet seem to have been considered in observational cosmology was also raised for discussion in the context of calculating variances (e.g., when quantifying backreaction terms): heavy tails in the distributions of physical quantities, such as those of the orbits of strongly interacting particles [80], ${ }^{\mathrm{g}}$ could lead to the need to study heavy-tailed distributions such as the Lévy distribution, which has a finite mode and median but an infinite mean and variance, [81] although truncated forms (which could occur if the Universe has a finite spatial volume) might be more realistic.

\section{Recent observational results}

The DE3 parallel session at MG14 included recent work that touches on several of the above issues ${ }^{\mathrm{h}}$. The observational disagreements with $\Lambda$ CDM listed above indicate a problem in the accuracy of the model, i.e., they are concerned with both systematic and random error, rather than a problem in the precision of the model, which is concerned with random error. Results indicating systematic errors that are normally unaccounted for in $\Lambda \mathrm{CDM}$ observational analyses, i.e., problems of accuracy, include Shanks' presentation of the "Local Hole" on a $100-200 h^{-1} \mathrm{Mpc}$ scale, Bolejko's discussion of a general-relativistic study of Hubble flow anisotropy on somewhat smaller scales, and Roukema's explanation of the recently found flexibility of the BAO scale. The other talks mostly presented observational results or projects that could potentially contribute to $\Lambda$ CDM's falsifiability in the coming decades, while not presently rejecting it. These include Mackenzie's presentation on the CMB Cold Spot and the ISW; Majerotto's discussion of the CBL test; Pisani's explanation of how to use 10-200 $h^{-1} \mathrm{Mpc}$ voids as a cosmological probe; and Kaminker's quasiperiodical statistical analysis of SDSS data.

A fundamental difficulty in observational cosmology follows from elementary geometry in a space that is approximately flat (or not too hyperbolic). Within a few $100 h^{-1} \mathrm{Mpc}$ from the observer, the spatial section contains very few $\left(100 h^{-1} \mathrm{Mpc}\right)^{3}$ volumes, leading to a high Poisson error in estimating any large-scale physical parameter, while at distances of $1 h^{-1} \mathrm{Gpc}$ or more, the Poisson noise (for a $100 h^{-1} \mathrm{Mpc}$-scale statistic) is more reasonable. This problem is commonly termed "cosmic variance". Recall that a general-relativistic interpretation of nearly empty spatial regions assigns a negative averaged curvature to these regions, which - if a Newtonian point of view is taken -implies ignorance of a physical variance in the curvature. (The cosmic variance problem also occurs for larger-scale statistics.) At the $100 h^{-1} \mathrm{Mpc}$ scale, there are two basic strategies: ignore the volume within a

\footnotetext{
${ }^{\mathrm{f}}$ See Ref. 79 for another recent summary of challenges to the standard model. g http://www.physik.fu-berlin.de/ kleinert/talks/CastTaiw1.pdf

$\mathrm{h}_{\mathrm{http}}$ ://www.icra.it/mg/mg14/; archived: http://www.webcitation.org/6c50Ydtut
} 
few $100 h^{-1} \mathrm{Mpc}$ of the observer and marginalise over a range of relativistically valid models of this volume, or impose a single theoretical model on the data analysis. Both approaches introduce a priori assumptions into the data analysis. The former would in principle be better, but the latter is what is used in practice.

Shanks presented new evidence that strengthens results that were suspected from faint galaxy number counts in the 1980s and were seriously quantified in the 2000s using the 2 Micron All-sky Survey (2MASS) [82], and by photometric followup of bright galaxies in the Two Degree Field Galaxy Redshift Survey (2dFGRS) [83]. These tentatively identified a $150-300 h^{-1} \mathrm{Mpc}$ scale underdensity with respect to more distant galaxies, including a celestial North-South asymmetry, dubbing it the "Local Hole". Whitbourn and Shanks confirmed this [84] by analysing 250,000 galaxy redshifts from the Six Degree Field Galaxy Survey (6dFGS) and the SDSS, using both their redshift distributions and number counts in comparison to the deeper Galaxy and Mass Assembly (GAMA) survey. They also made peculiar velocity maps that rejected the possibility of the local $150 h^{-1} \mathrm{Mpc}$ diameter region being at rest in the CMB frame at $4 \sigma$. In as yet unpublished results together with Whitbourn, Shanks explains how clustering-independent luminosity function analysis, an independent galaxy survey, and a complete X-ray cluster survey corroborate their earlier results.

Studies of the pattern of galaxies' peculiar velocities - defined by subtraction of either a naïve (strictly linear) Hubble law [85], or a third-order Taylor expansion or analytically exact expression for the redshift-distance relation for an FLRW model - have led to decades of community debate about whether cosmic flows are compatible or incompatible with $\Lambda$ CDM e.g., Refs. 71, 86, 87 and references therein). Bolejko's new work with Wiltshire and Nazer [76] provides a fresh look at this model-dependent observational debate. They have performed numerical simulations by ray-tracing in exact Szekeres models with inhomogeneous structures which match as closely as possible the dominant inhomogeneities observed on scales $\lesssim 80 h^{-1} \mathrm{Mpc}$, while asymptotically matching a Planck-satellite normalized $\Lambda \mathrm{CDM}$ model on larger scales. These simulations are quantitatively constrained by the requirement that they be consistent with the observed CMB anisotropies, while incorporating a nonkinematic CMB dipole component. The dipole and quadrupole variations of the local Hubble expansion are then quantitatively compared to those of the COMPOSITE sample data as studied earlier in Ref. 74. It is found that the Szekeres model is able to more closely model observational features than either a FLRW model with kinematic boost from the LG to CMB frame, or Newtonian $N$-body simulations. In fact, the latter models are rejected at more than the $2 \sigma$ level.

Either an increased observational confidence in the existence of the Local Hole or a preference for the Szekeres model over the standard model on a smaller scales would separately or jointly imply that systematic errors need to be taken into account for many, though not all, observational analyses on $1-20 h^{-1} \mathrm{Gpc}$ scales. An- 
gles and distances to objects beyond $100-200 h^{-1} \mathrm{Mpc}$ depend on correctly propagating light through the geometry on scales inside of $100-200 h^{-1} \mathrm{Mpc}$. This point is not always appreciated, with the incorrect perception that inferences of FLRW cosmological parameters from CMB observations are independent of the assumed foreground geometry, whether this is on a tens of megaparsecs scale or greater. The possibility of a nonkinematic contribution to the CMB dipole is actually directly relevant to the question of large-angle anomalies, and a future challenge is to investigate this directly in the CMB map-making procedures.

On a scale up to a little less than $1 h^{-1} \mathrm{Gpc}$, Roukema presented recent SDSS DR7 work with Buchert, Fujii and Ostrowski [53] extending previous work [52] that showed that the $\approx 105 h^{-1} \mathrm{Mpc} \mathrm{BAO}$ peak location, normally thought of as a comoving standard ruler, is shrunk by a fraction of $6 \%$ across SDSS DR7 superclusters. This is expected from scalar averaging, which can be seen as a generalrelativistically more careful generalisation of the FLRW model [88-90], but has not been predicted in the $\Lambda$ CDM model. The expected redshift-dependent shift (rather than an environment-dependent shift) has been modelled [91,92], but the expected shift is tiny, e.g., $<0.3 \%$ [92]. If the environment dependence were genuinely an environment-dependent effect, then it should strengthen as the required overlap between galaxy pairs and superclusters increased. This is indeed the case, as was shown in the recent work presented at the meeting, with the shift increasing up to $10 \%$ of the peak location [53]. This presently only qualifies as a qualitative inconsistency with $\Lambda$ CDM. Future work should confirm whether or not the environmentdependent BAO peak location shift is quantitatively consistent with $\Lambda$ CDM.

The other observational presentations at the DE3 session concerned ongoing projects that do not presently reject the $\Lambda$ CDM model, but could potentially help to separate the $\Lambda$ CDM model from relativistic inhomogeneous models and explicitly non-GR models. Mackenzie presented work he is doing with Shanks on the ISW effect by cross-correlating the LRG distribution using photometric redshifts from a $\sim 4700 \mathrm{deg}^{2}$ photometric southern optical survey with Planck Surveyor CMB maps. He plans to test the nature of the $\sim 400 h^{-1} \mathrm{Mpc}$ supervoid [93] in front of the CMB Cold Spot and whether the two are physically related; see also Ref. 94.

Majerotto presented work together with Sapone and Nesseris [95] who applied the Clarkson, Bassett and Lu test $[38,96]$ using eight $H(z)$ cosmic chronometer estimates [97] (age of oldest passively evolving red galaxies at any given redshift $z[98])$ and a recent compilation of supernovae type Ia redshift-magnitude estimates. This test should distinguish some classes of relativistic or other non-FLRW models from the FLRW model. The uncertainties with the presently used data sets were found to be large, with the FLRW model being found to be consistent with the data. What is particularly interesting for the coming decade are predictions for redshift evolution of $\widehat{\Omega}_{k}$, the average curvature parameter, from telescopes such as Euclid. Majerotto showed that statistically homogeneous and isotropic general-relativistic cosmological models in which the expansion rate history is observationally realistic, 
including the Timescape $[40,99,100]$ and Tardis [45] models, have $\widehat{\Omega}_{k}(z)$ relations that should be observationally distinguishable from $\Lambda \mathrm{CDM}$ to high significance by Euclid [95]. Both the models, as in the case of template metrics that match the supernovae type Ia distance-modulus-redshift relation, i.e., that of Ref. 39 and the virialisation approximation [101], have an average curvature parameter which evolves from a small value to a strongly negative average effective curvature today. In Ref. 39 it is also concluded that this curvature evolution is detectable by Euclid.

It has been known for at least three decades [102] that cosmic voids exist on scales of tens of megaparsecs. These have recently become the subject of systematic study [59,63,64], especially since the release of the SDSS. Voids on scales ranging from $\sim 10-200 h^{-1} \mathrm{Mpc}$ are now the subject of intense debate, linked to the fundamental disadvantage in detecting underdensities as opposed to overdensities [103-107]. With the tracers being (for these purposes) essentially point objects rather than a continuous fluid, the Poisson error on an "average" galaxy density of, e.g., 0.2 galaxies in a given number of cubic megaparsecs is proportionally huge in comparison to studies of overdense regions. It is thus likely that the debate will require considerable attention from the community before converging in regard to optimal strategies for catalogue analysis. Pisani presented recent work on some of these questions, including the role of peculiar velocities with respect to an assumed FLRW model, and predictions for how well void analysis will help in parametrising cosmological parameters from the upcoming Euclid and WFIRST missions, provided that the FLRW model is assumed.

Kaminker presented his work with Ryabinkov [108] based on spectroscopic redshifts of 52,683 brightest cluster galaxies (BCGs) over the interval $0.044 \leq z \leq 0.78$ from the SDSS DR7 [109] and 32,840 Mg II absorption line systems (ALSes) over $0.37 \leq z \leq 2.28$ [110]. The aim was to analyse structure, separately using onedimensional Fourier analysis and two-point auto-correlation functions, i.e., in effect, projecting the full solid angle of the SDSS into a single pencil beam. For the $\Lambda \mathrm{CDM}$ model with $\Omega_{\mathrm{m} 0}=0.32$, the distance ranges of the BCGs range from $130-1900 h^{-1} \mathrm{Mpc}$, while the Mg II ALSes lie from $1000-3800 h^{-1} \mathrm{Mpc}$ from the observer. Since the diameter of the SDSS main observing region is roughly a radian, this implies that typically 10-20 BAO peak scale regions cross the observing cone of these two subsamples. The BAO peak itself is only a small bump above the smooth part of the two-point auto-correlation function. Unless there is a fair amount of phase alignment, the tangential projection together of ten to twenty regions of that size prior to calculation of either a Fourier spectrum or a correlation function in the radial direction should lead to an obscuring (convolution) of the bump, leaving a smoothed out version of the main constituent of the power spectrum of primordial density perturbations. However, Ryabinkov and Kaminker removed much of the large-scale power by fitting a smooth function to the redshift distribution for each sub-sample, so the remaining power should correspond to scales less than a few hundred megaparsecs. 
The results, calculated for a $\Lambda \mathrm{CDM}$ model with $\Omega_{\mathrm{m} 0}=0.25$, showed an im-

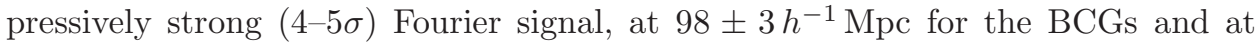
$101 \pm 2 h^{-1} \mathrm{Mpc}$ for the Mg II ALSes [108], somewhat below the standard BAO peak scale. Given that typically 10-20 BAO peak scale regions are tangentially mixed together in each of these sub-samples, this could indicate that the phases of large-scale structure on the BAO scale in the SDSS are somewhat aligned, violating the usual assumption of random phases of the primordial density perturbations. This would be a potentially very interesting test of $\Lambda \mathrm{CDM}$, yielding a much stronger result than the older pencil beam results that showed a $128 h^{-1} \mathrm{Mpc}$ periodicity [111], which in Newtonian structure formation models - that remove small-scale power and introduce a Lagrangian biasing of the density distribution - was shown to be a natural outcome [112], while other work using $N$-body simulations found it to be unlikely at the $0.1 \%$ level [113]. Full-scale modelling with $N$-body simulations to see if the Ryabinkov and Kaminker results are compatible with a standard power spectrum, including BAOs and random phases, would be highly justified.

\section{Conclusion}

We have not attempted a Bayesian summary of all available observational evidence for and against the $\Lambda \mathrm{CDM}$ model. It is uncontroversially a good fit to many observations, despite assuming a prior, Newtonian structure formation, which has an extremely low (Bayesian) likelihood compared to general relativity in the only regimes in which both theories can be directly tested. Pending Bayesian analyses that include the differences between Newtonian and relativistic approaches, a frequentist approach to many of the individual tests that presently reject the $\Lambda$ CDM to high significance, and to those that may potentially reject it, is justified. The number of disagreements, including those discussed at MG14 - whether labelled "rejections", "inconsistencies", "anomalies" or "tensions" — cannot be ignored.

Many predictions of the FLRW models are already well known, and will continue to be tested in future astronomical missions such as Euclid. In contrast, predictions for general-relativistic cosmological models that take into account structure formation are still in their infancy. However, this field of research is developing rapidly, and the initially published predictions are likely to be made more robust in the coming years. In particular, rather than seeking ways to reconcile the $\Lambda \mathrm{CDM}$ model with tests that reject it, we feel that it is important to think about the observational jigsaw puzzles and come up with new predictions that are relativistically realistic. Relaxing some of the physical restrictions of the $\Lambda \mathrm{CDM}$ model, such as a rigid vanishing spatial curvature on all scales and throughout cosmic history, may lead to a consensus in the new modelling efforts that enrich cosmology as a physical science.

\section{Acknowledgments}

We would like to thank Mauro Carfora, George Ellis, Edward W. "Rocky" Kolb, Malcolm MacCallum, Jan Ostrowski, Syksy Räsänen, Lars Andersson, Krzysztof 
Bolejko and other colleagues at MG14 for constructive discussions. The work of TB was conducted within the "Lyon Institute of Origins" under grant ANR-10-LABX66. TB and BFR acknowledge support for a part of this project from the HECOLS International Associated Laboratory, supported in part by the National Science Centre, Poland, grant DEC-2013/08/M/ST9/00664, and for a part of the project under grant 2014/13/B/ST9/00845 of the National Science Centre, Poland, and BFR acknowledges visiting support from CRALyon. AAC acknowledges support from NSERC.

\section{References}

1. S. Abdi, Bonferroni and Sidak corrections for multiple comparisons, in Encyclopedia of Measurement and Statistics, (Thousand Oaks, Sage, USA, 2007), Sage, USA, http: //www.utdallas. edu/ herve/Abdi-Bonferroni2007-pretty . pdf.

2. J. M. Weisberg, D. J. Nice and J. H. Taylor, Astrophys. J. 722 (2010) 1030, arXiv:1011.0718 [astro-ph.GA].

3. R. A. Hulse and J. H. Taylor, Astrophys. J. Lett. 195 (1975) L51.

4. R. H. Cyburt, B. D. Fields and K. A. Olive, Journ. Cosm. Astr. Phys. 11 (2008) 12, arXiv:0808.2818.

5. R. H. Cyburt, J. Ellis, B. D. Fields, F. Luo, K. A. Olive and V. C. Spanos, Journ. Cosm. Astr. Phys. 5 (2013) 14, arXiv:1303.0574 [astro-ph.CO].

6. P. A. R. Ade, N. Aghanim, C. Armitage-Caplan, M. Arnaud, M. Ashdown, F. AtrioBarandela, J. Aumont, C. Baccigalupi et al., Astron. Astroph. 571 (2014) A23, arXiv:1303.5083.

7. P. A. R. Ade, N. Aghanim, Y. Akrami, P. K. Aluri, M. Arnaud, M. Ashdown, J. Aumont, C. Baccigalupi et al., ArXiv e-prints (2015) arXiv:1506.07135.

8. D. N. Spergel, L. Verde, H. V. Peiris, E. Komatsu, M. R. Nolta, C. L. Bennett, M. Halpern, G. Hinshaw et al., Astrophys. J. Supp. 148 (2003) 175, astro-ph/0302209.

9. C. J. Copi, D. Huterer, D. J. Schwarz and G. D. Starkman, Phys. Rev. D 75 (2007) 023507, astro-ph/0605135.

10. A. Gruppuso, Mon. Not. Roy. Astr. Soc. 437 (2014) 2076, arXiv:1310.2822 [astro-ph.CO].

11. C. J. Copi, D. Huterer, D. J. Schwarz and G. D. Starkman, Mon. Not. Roy. Astr. Soc. 451 (2015) 2978, arXiv:1310.3831 [astro-ph.C0].

12. A. A. Starobinsky, J. Exper. Theor. Phys. Lett. 57 (1993) 622.

13. D. Stevens, D. Scott and J. Silk, Phys. Rev. Lett. 71 (1993) 20.

14. R. Aurich, Class. Quant. Gravit. 25 (2008) 225017, 0803.2130.

15. J. Luminet, J. R. Weeks, A. Riazuelo, R. Lehoucq and J. Uzan, Nature 425 (2003) 593, arXiv:astro-ph/0310253.

16. B. F. Roukema, Z. Buliński, A. Szaniewska and N. E. Gaudin, Astron. Astroph. 486 (2008) 55, 0801.0006.

17. B. F. Roukema and T. A. Kazimierczak, Astron. Astroph. 533 (2011) A11, arXiv:1106.0727 [astro-ph.CO].

18. B. F. Roukema, M. J. France, T. A. Kazimierczak and T. Buchert, Mon. Not. Roy. Astr. Soc. 437 (2014) 1096, arXiv: 1302.4425 [astro-ph.Co].

19. R. Aurich and S. Lustig, Mon. Not. Roy. Astr. Soc. (2013) arXiv:1303.4226 [astro-ph.CO].

20. P. A. R. Ade, N. Aghanim, C. Armitage-Caplan, M. Arnaud, M. Ashdown, F. Atrio- 
Barandela, J. Aumont, C. Baccigalupi et al., Astron. Astroph. 571 (2014) A26, arXiv:1303.5086.

21. H. K. Eriksen, F. K. Hansen, A. J. Banday, K. M. Górski and P. B. Lilje, Astrophys. J. 605 (2004) 14, astro-ph/0307507.

22. J. Hoftuft, H. K. Eriksen, A. J. Banday, K. M. Górski, F. K. Hansen and P. B. Lilje, Astrophys. J. 699 (2009) 985, arXiv:0903.1229 [astro-ph.CO].

23. P. A. R. Ade, N. Aghanim, C. Armitage-Caplan, M. Arnaud, M. Ashdown, F. AtrioBarandela, J. Aumont, C. Baccigalupi et al., Astron. Astroph. 571 (2014) A16, arXiv:1303.5076 [astro-ph.CO].

24. S. Mukherjee, P. K. Aluri, S. Das, S. Shaikh and T. Souradeep, ArXiv e-prints (2015) arXiv: 1510.00154.

25. M. Tegmark, A. de Oliveira-Costa and A. Hamilton, Phys. Rev. D 68 (2003) 123523, astro-ph/0302496.

26. C. J. Copi, D. Huterer, D. J. Schwarz and G. D. Starkman, Mon. Not. Roy. Astr. Soc. 449 (2015) 3458, arXiv:1311.4562 [astro-ph.C0].

27. B. R. Granett, M. C. Neyrinck and I. Szapudi, Astrophys. J. Lett. 683 (2008) L99, arXiv:0805.3695.

28. S. Flender, S. Hotchkiss and S. Nadathur, Journ. Cosm. Astr. Phys. 2 (2013) 13, arXiv:1212.0776 [astro-ph.CO].

29. A. Wiegand, T. Buchert and M. Ostermann, Mon. Not. Roy. Astr. Soc. 443 (2014) 241, arXiv:1311.3661 [astro-ph.C0].

30. R. A. Battye, T. Charnock and A. Moss, Phys. Rev. D 91 (2015) 103508, arXiv:1409.2769.

31. N. MacCrann, J. Zuntz, S. Bridle, B. Jain and M. R. Becker, Mon. Not. Roy. Astr. Soc. 451 (2015) 2877, arXiv:1408.4742.

32. T. Delubac, J. E. Bautista, N. G. Busca, J. Rich, D. Kirkby, S. Bailey, A. FontRibera, A. Slosar et al., Astron. Astroph. 574 (2015) A59, arXiv:1404.1801.

33. C. Blake, S. Brough, M. Colless, C. Contreras, W. Couch, S. Croom, D. Croton, T. M. Davis et al., Mon. Not. Roy. Astr. Soc. 425 (2012) 405, arXiv:1204.3674 [astro-ph.CO].

34. C.-H. Chuang, F. Prada, F. Beutler, D. J. Eisenstein, S. Escoffier, S. Ho, J.-P. Kneib, M. Manera et al., ArXiv e-prints (2013) arXiv:1312.4889 [astro-ph.Co].

35. V. Salvatelli, N. Said, M. Bruni, A. Melchiorri and D. Wands, Physical Review Letters 113 (2014) 181301, arXiv:1406.7297.

36. F. Melia, Astron. J. 147 (2014) 120, arXiv:1403.0908 [astro-ph.CO].

37. P. Kroupa, Proc. Astr. Soc. Austr. 29 (2012) 395, arXiv:1204.2546.

38. C. Clarkson, B. Bassett and T. H.-C. Lu, Physical Review Letters 101 (2008) 011301, arXiv:0712.3457.

39. J. Larena, J.-M. Alimi, T. Buchert, M. Kunz and P.-S. Corasaniti, Phys. Rev. D 79 (2009) 083011, arXiv:0808.1161.

40. D. L. Wiltshire, Phys. Rev. D 80 (2009) 123512, arXiv:0909.0749 [astro-ph.Co].

41. S. Räsänen, Journ. Cosm. Astr. Phys. 2 (2009) 11, arXiv:0812.2872.

42. A. Shafieloo and C. Clarkson, Phys. Rev. D 81 (2010) 083537, arXiv:0911.4858 [astro-ph.CO].

43. S. Räsänen, Journ. Cosm. Astr. Phys. 3 (2010) 18, arXiv:0912.3370 [astro-ph.Co].

44. C. Boehm and S. Räsänen, Journ. Cosm. Astr. Phys. 9 (2013) 003, arXiv:1305.7139 [astro-ph.CO].

45. M. Lavinto, S. Räsänen and S. J. Szybka, Journ. Cosm. Astr. Phys. 12 (2013) 51, arXiv:1308.6731 [astro-ph.C0]. 
46. D. L. Wiltshire, Cosmic structure, averaging and dark energy, in Proc. XVth Brazilian School of Cosmology and Gravitation, eds. Novello, M. and Perez Bergliaffa, S. E. (Cambridge: Cambridge Scientific Publishers, 2014), pp. 203-244. arXiv:1311.3787 [astro-ph.C0].

47. M. Chiesa, D. Maino and E. Majerotto, Journ. Cosm. Astr. Phys. 12 (2014) 49, arXiv: 1405.7911.

48. A. Gabrielli, F. Sylos Labini, M. Joyce and L. Pietronero, Statistical physics for cosmic structures (Berlin: Springer, 2005).

49. D. W. Hogg, D. J. Eisenstein, M. R. Blanton, N. A. Bahcall, J. Brinkmann, J. E. Gunn and D. P. Schneider, Astrophys. J. 624 (2005) 54, astro-ph/0411197.

50. M. I. Scrimgeour, T. Davis, C. Blake, J. B. James, G. B. Poole, L. StaveleySmith, S. Brough, M. Colless et al., Mon. Not. Roy. Astr. Soc. 425 (2012) 116, arXiv:1205.6812.

51. M. Kerscher, K. Mecke, J. Schmalzing, C. Beisbart, T. Buchert and H. Wagner, Astron. Astroph. 373 (2001) 1, arXiv:astro-ph/0101238.

52. B. F. Roukema, T. Buchert, J. J. Ostrowski and M. J. France, Mon. Not. Roy. Astr. Soc. 448 (2015) 1660, arXiv:1410.1687.

53. B. F. Roukema, T. Buchert, H. Fujii and J. J. Ostrowski, Mon. Not. Roy. Astr. Soc. 456 (2016) L45, arXiv:1506.05478.

54. F. Sylos Labini, N. L. Vasilyev, L. Pietronero and Y. V. Baryshev, Europhys. Lett. 86 (2009) 49001, 0805.1132.

55. S. R. Green and R. M. Wald, Class. Quant. Gravit. 31 (2014) 234003, arXiv:1407.8084 [gr-qc].

56. S. R. Green and R. M. Wald, Phys. Rev. D 83 (2011) 084020,

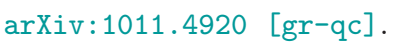

57. T. Buchert, M. Carfora, G. F. R. Ellis, E. W. Kolb, M. A. H. MacCallum, J. J. Ostrowski, S. Räsänen, B. F. Roukema, L. Andersson, A. A. Coley and D. L. Wiltshire, Class. Quant. Gravit. 32 (2015) 215021, arXiv:1505.07800 [gr-qc].

58. J. J. Ostrowski and B. F. Roukema, On the Green and Wald formalism, in Proceedings of the Fourteenth Marcel Grossmann Meeting on General Relativity, eds. M. Bianchi, R. T. Jantzen and R. Ruffini (Singapore: World Scientific, 2016). arXiv:1512.02947 [gr-qc].

59. D. C. Pan, M. S. Vogeley, F. Hoyle, Y.-Y. Choi and C. Park, Mon. Not. Roy. Astr. Soc. 421 (2012) 926, arXiv:1103.4156 [astro-ph.CO].

60. A. V. Tikhonov and I. D. Karachentsev, Astrophys. J. 653 (2006) 969, arXiv:astro-ph/0609109.

61. J. M. Colberg, F. Pearce, C. Foster, E. Platen, R. Brunino, M. Neyrinck, S. Basilakos, A. Fairall et al., Mon. Not. Roy. Astr. Soc. 387 (2008) 933, arXiv:0803.0918.

62. M. Cautun, R. van de Weygaert, B. J. T. Jones and C. S. Frenk, Understanding the cosmic web, in The Zeldovich Universe: Genesis and Growth of the Cosmic Web, eds. R. van de Weygaert, S. Shandarin, E. Saar and J. Einasto, IAU Symposium, Vol. 308 (2015). arXiv: 1501.01306.

63. F. Hoyle and M. S. Vogeley, Astrophys. J. 566 (2002) 641, arXiv: astro-ph/0109357.

64. F. Hoyle and M. S. Vogeley, Astrophys. J. 607 (2004) 751, arXiv: astro-ph/0312533.

65. J. Adamek, D. Daverio, R. Durrer and M. Kunz, ArXiv e-prints (2015) arXiv:1509.01699.

66. J. B. Mertens, J. T. Giblin, Jr and G. D. Starkman, ArXiv e-prints (2015) arXiv: 1511.01106 [gr-qc].

67. J. T. Giblin, Jr, J. B. Mertens and G. D. Starkman, ArXiv e-prints (2015) arXiv:1511.01105 [gr-qc]. 
68. E. Bentivegna and M. Bruni, ArXiv e-prints (2015) arXiv:1511.05124 [gr-qc].

69. M. Bruni et al., in Proceedings of the Fourteenth Marcel Grossmann Meeting on General Relativity, eds. M. Bianchi, R. T. Jantzen and R. Ruffini (Singapore: World Scientific, 2016), in preparation.

70. R. B. Tully, E. J. Shaya, I. D. Karachentsev, H. M. Courtois, D. D. Kocevski, L. Rizzi and A. Peel, Astrophys. J. 676 (2008) 184, arXiv:0705.4139.

71. G. Lavaux, R. B. Tully, R. Mohayaee and S. Colombi, Astrophys. J. 709 (2010) 483, arXiv:0810.3658.

72. M. Bilicki, M. Chodorowski, T. Jarrett and G. A. Mamon, Astrophys. J. 741 (2011) 31, arXiv:1102.4356.

73. S. Hess and F.-S. Kitaura, Mon. Not. Roy. Astr. Soc. 456 (2016) 4247, arXiv:1412.7310.

74. D. L. Wiltshire, P. R. Smale, T. Mattsson and R. Watkins, Phys. Rev. D 88 (2013) 083529, arXiv:1201.5371 [astro-ph.CO].

75. J. H. McKay and D. L. Wiltshire, Mon. Not. Roy. Astr. Soc. (2016) in press, doi:10.1093/mnras/stw128, arXiv: 1503.04192.

76. K. Bolejko, M. A. Nazer and D. L. Wiltshire, ArXiv e-prints (2015) arXiv:1512.07364.

77. N. Aghanim, C. Armitage-Caplan, M. Arnaud, M. Ashdown, F. Atrio-Barandela, J. Aumont, C. Baccigalupi, A. J. Banday and et al., Astron. Astroph. 571 (2014) A27, arXiv:1303.5087.

78. M. Rubart and D. J. Schwarz, Astron. Astroph. 555 (2013) A117, arXiv:1301.5559 [astro-ph.CO].

79. P. Bull, Y. Akrami, J. Adamek, T. Baker, E. Bellini, J. Beltrán Jiménez, E. Bentivegna, S. Camera et al., ArXiv e-prints (2015) arXiv:1512.05356.

80. H. Kleinert, EPL (Europhysics Letters) $100 \quad$ (2012) 10001, arXiv:1210.2630 [quant-ph].

81. B. Podobnik, P. C. Ivanov, Y. Lee, A. Chessa and H. E. Stanley, EPL (Europhysics Letters) $\mathbf{5 0}$ (2000) 711, cond-mat/9910433.

82. W. J. Frith, G. S. Busswell, R. Fong, N. Metcalfe and T. Shanks, Mon. Not. Roy. Astr. Soc. 345 (2003) 1049, astro-ph/0302331.

83. G. S. Busswell, T. Shanks, W. J. Frith, P. J. Outram, N. Metcalfe and R. Fong, Mon. Not. Roy. Astr. Soc. 354 (2004) 991, astro-ph/0302330.

84. J. R. Whitbourn and T. Shanks, Mon. Not. Roy. Astr. Soc. 437 (2014) 2146, arXiv:1307.4405.

85. T. M. Davis and M. I. Scrimgeour, Mon. Not. Roy. Astr. Soc. 442 (2014) 1117, arXiv:1405.0105.

86. K. Tomita, Astrophys. J. $\mathbf{5 2 9}$ (2000) 26, astro-ph/9905278.

87. C. M. Springob, T. Hong, L. Staveley-Smith, K. L. Masters, L. M. Macri, B. S. Koribalski, D. H. Jones, T. H. Jarrett et al., Mon. Not. Roy. Astr. Soc. 456 (2016) 1886, arXiv:1511.04849.

88. T. Buchert, Gen. Rel. Gravit. 32 (2000) 105, gr-qc/9906015.

89. T. Buchert, Gen. Rel. Gravit. 33 (2001) 1381, gr-qc/0102049.

90. T. Buchert, Gen. Rel. Gravit. 40 (2008) 467, arXiv:0707.2153 [gr-qc].

91. V. Desjacques, M. Crocce, R. Scoccimarro and R. K. Sheth, Phys. Rev. D 82 (2010) 103529, arXiv:1009.3449 [astro-ph.Co].

92. B. D. Sherwin and M. Zaldarriaga, Phys. Rev. D 85 (2012) 103523, arXiv:1202.3998 [astro-ph.CO].

93. I. Szapudi, A. Kovács, B. R. Granett, Z. Frei, J. Silk, W. Burgett, S. Cole, P. W. Draper et al., Mon. Not. Roy. Astr. Soc. 450 (2015) 288, arXiv:1405.1566. 
94. A. Enea Romano, D. Cornejo and L. E. Campusano, ArXiv e-prints (2015) arXiv: 1509.01879.

95. D. Sapone, E. Majerotto and S. Nesseris, Phys. Rev. D 90 (2014) 023012, arXiv:1402.2236.

96. C. Clarkson, Comptes Rendus Physique 13 (2012) 682, arXiv:1204.5505.

97. M. Moresco, A. Cimatti, R. Jimenez, L. Pozzetti, G. Zamorani, M. Bolzonella, J. Dunlop, F. Lamareille et al., Journ. Cosm. Astr. Phys. 8 (2012) 6, arXiv:1201.3609 [astro-ph.CO].

98. R. Jimenez and A. Loeb, Astrophys. J. 573 (2002) 37, astro-ph/0106145.

99. J. A. G. Duley, M. A. Nazer and D. L. Wiltshire, Class. Quant. Gravit. 30 (2013) 175006, arXiv:1306.3208 [astro-ph.C0].

100. M. A. Nazer and D. L. Wiltshire, Phys. Rev. D 91 (2015) 063519, arXiv:1410.3470.

101. B. F. Roukema, J. J. Ostrowski and T. Buchert, Journ. Cosm. Astr. Phys. 10 (2013) 043, arXiv:1303.4444 [astro-ph.C0].

102. V. de Lapparent, M. J. Geller and J. P. Huchra, Astrophys. J. Lett. 302 (1986) L1.

103. S. Nadathur and S. Hotchkiss, Mon. Not. Roy. Astr. Soc. 440 (2014) 1248, arXiv:1310.2791 [astro-ph.CO].

104. P. M. Sutter, G. Lavaux, B. D. Wandelt, D. H. Weinberg, M. S. Warren and A. Pisani, Mon. Not. Roy. Astr. Soc. 442 (2014) 3127, arXiv:1310.7155.

105. A. Pisani, G. Lavaux, P. M. Sutter and B. D. Wandelt, Mon. Not. Roy. Astr. Soc. 443 (2014) 3238, arXiv:1306.3052.

106. A. Pisani, P. M. Sutter, N. Hamaus, E. Alizadeh, R. Biswas, B. D. Wandelt and C. M. Hirata, Phys. Rev. D 92 (2015) 083531, arXiv:1503.07690.

107. A. Pisani, P. M. Sutter and B. D. Wandelt, ArXiv e-prints (2015) arXiv:1506.07982.

108. A. I. Ryabinkov and A. D. Kaminker, Mon. Not. Roy. Astr. Soc. 440 (2014) 2388, arXiv:1402.5597.

109. Z. L. Wen and J. L. Han, Updated catalog of 132,684 galaxy clusters and evolution of brightest cluster galaxies, in IAU Symposium, eds. D. Thomas, A. Pasquali and I. Ferreras, IAU Symposium, Vol. 295 (2013), pp. 188-188. arXiv:1301.0871.

110. G. Zhu and B. Ménard, Astrophys. J. $\mathbf{7 7 0}$ (2013) 130, arXiv:1211.6215.

111. T. J. Broadhurst, R. S. Ellis, D. C. Koo and A. S. Szalay, Nature 343 (1990) 726.

112. A. G. Weiss and T. Buchert, Astron. Astroph. 274 (1993) 1.

113. N. Yoshida, J. Colberg, S. D. M. White, A. E. Evrard, T. J. MacFarland, H. M. P. Couchman, A. Jenkins, C. S. Frenk et al., Mon. Not. Roy. Astr. Soc. 325 (2001) 803, astro-ph/0011212. 